\title{
IEEE Wireless LAN Capacity in Multicell Environments with Rate Adaptation
}

\author{
Eduard Garcia, Elena Lopez-Aguilera, Rafael Vidal and Josep Paradells \\ Wireless Networks Group, Telematics Engineering Dept., Technical University of Catalonia (UPC)
}

\begin{abstract}
Since the advent of the first IEEE 802.11 standard, many research efforts have been spent on evaluating different aspects of the specification. In this paper, we present a new method to predict the capacity of a multicell IEEE 802.11 network. The mechanism takes the effect of co-channel and adjacent channel interference into account. In addition, the study of a common rate adaptation algorithm is included. When the effect of rate adaptation is considered within the throughput computation, the results provided by our algorithm are closer to the measurements obtained in a real scenario. To the best of our knowledge, this paper presents the first analytical study of throughput performance including both types of interferences and the effect of bit rate adaptation.
\end{abstract}

\section{INTRODUCTION}

Having tools able to predict the performance of Wireless Local Area Networks (WLANs) is essential to evaluate the quality of a design before its deployment. A common option is the use of simulators, but aside from the expense involved, the way they model interferences in multicell environments is not accurate due to simplifications in the physical layer - cochannel and adjacent channel interference emulation does not behave as observed in real testbeds. An alternative lies in the use of analytical models, which also make significant assumptions. However, they are faster in providing a reliable approximation of a network's performance and the results are precise enough to allow the objective evaluation of different WLAN designs. Capacity estimations can then be used as the metric to compare different frequency channel schemes, different power allocations, user-AP (Access Point) associations, etc. In other words, capacity estimations can assist many radio resource management (RRM) mechanisms that are in charge of dynamically tuning different WLAN parameters in order to optimize its performance. Our aim is therefore to develop a straightforward algorithm that is able to provide capacity estimations in a timely manner so that they can be used by different RRM mechanisms as an immediate measure of the potential performance of a WLAN.

In the present paper, a new algorithm is studied that allows the capacity calculation for multicell WLANs, by adapting the known Bianchi's model [1]. This model was used to provide the saturation throughput in a single WLAN cell where there are neither transmission errors nor hidden terminals. Our adaptation includes not only transmission errors, which has been already studied in the literature, but also the effect of co-channel interference. The way the nodes of a WLAN share the medium is similar to an Ethernet segment. A Carrier Sense Multiple Access with Collision Avoidance (CSMA/CA) mechanism is used as Medium Access Control (MAC) scheme. This makes the study of interferences in WLAN IEEE 802.11 quite different from

This research work has been funded by DURSI, ERDF, the Spanish Government through project TEC2006-0454 and the i2CAT foundation. what is done in other radio networks, due to the particular influence of interference produced by cells using the same channel (co-channel interference). In a cell suffering only cochannel interference, even though there is no traffic on it, the nodes may also defer their transmissions if when sensing the medium, they detect other signals above a certain threshold in their working channel - these terminals are known as exposed nodes.

Furthermore, most of the IEEE 802.11 implementations found on the market today apply proprietary link adaptation schemes, in order to select the best modulation (physical rate) according to the current channel conditions. These algorithms are, in general, not available to the public, thus hampering the inclusion of rate adaptation in realistic analytical throughput models. For these reasons, we also introduce in the resulting formulation, the effect of a simple link adaptation algorithm. We have to note that the heuristic we propose is intended only for modeling purposes and not for implementation. Our contribution is therefore twofold: to the best of our knowledge, this paper presents the first analytical study of throughput performance including both types of interferences, co-channel and adjacent channel, and the effect of bit rate adaptation.

The rest of the paper is organized as follows: in Section II, we give a brief introduction of IEEE 802.11 MAC standard. Section III reviews the basic formulation to compute the throughput of IEEE 802.11 WLANs given in the literature, and also presents our proposal to include co-channel interference. Section IV provides a state-of-the-art overview regarding rate adaptation and a simple heuristic algorithm is analyzed. The evaluation of our formulation is given in Section V. Finally, concluding remarks are in Section VI.

\section{IEEE 802.11 PROTOCOLS}

Since the definition of the first IEEE 802.11 standard for WLANs, several variants have appeared that increase the bit rate. However, the $M A C$ working procedure has remained the same. The IEEE 802.11 MAC procedure provides two operating modes: Distributed Coordination Function (DCF) and Point Coordination Function (PCF). The DCF uses the contention $M A C$ algorithm $C S M A / C A$, whereas the $P C F$ offers contention free access. The two modes are used alternately in time.

The $D C F$ works as follows. Before initiating a transmission, a station senses the channel to determine whether it is busy. If the medium is sensed idle during a period of time called Distributed Interframe Space (DIFS), the station is allowed to transmit. If the medium is sensed busy, the transmission is delayed until the channel is idle again. A slotted binary exponential backoff interval is uniformly chosen in $[0, C W-1]$, where $C W$ is the contention window. The backoff timer is decreased as long as the 
channel is sensed idle, stopped when a transmission is in progress, and reactivated when the channel is sensed idle again for more than DIFS. When the backoff timer expires, the station starts transmitting. After each data frame successfully received, the receiver transmits an acknowledgment frame (ACK) after a Short Interframe Space (SIFS) period. The value of $C W$ is set to its minimum value, $C W_{\text {min }}$, in the first transmission attempt, and ascends integer powers of two at each retransmission, up to a pre-determined value $C W_{\max }$.

The protocol described above is called basic or two-way handshaking mechanism. In addition, the specification also contains a four-way frame's exchange protocol called RTS/CTS mechanism, which works as follows. A station gains channel access through the contention process described previously, and sends a special frame called Request to Send (RTS), instead of the actual data frame. In response to that, the receiver sends a Clear to Send (CTS) frame after a SIFS interval. Subsequently, the requesting station is allowed to start the data frame's transmission after a SIFS period. The main objective of RTS/CTS handshake is the resolution of the hidden terminal problem. The mechanism is also employed to minimize the lost periods caused by collisions - the RTS frame is much shorter than data fames.

Finally, the IEEE 802.11 DCF MAC protocol supports two kinds of Basic Service Set (BSS): the independent BSS, known as ad-hoc networks, which have no connection to wired networks, and the infrastructure $B S S$, which contains an AP connected to the wired network. The second kind of BSS assimilates to cellular networks with base stations.

\section{SATURATiOn Throughput CALCUlation}

To carry out our evaluation we have performed a mathematical analysis based on Bianchi's model [1]. Original Bianchi's model allows an accurate evaluation of the saturation throughput of IEEE 802.11 DCF networks under the assumption of ideal channel conditions and considering unlimited retransmissions, by employing a Markov chain. It concludes with the following expression for the saturation throughput:

$$
S_{t}=\frac{P_{t r} P_{S} E_{p}}{E_{S}}
$$

where $S_{t}$ is the saturation throughput defined as the fraction of time the channel is used to successfully transmit payload bits, $E_{s}$ is the average length of a renewal interval, defined as the time between two consecutive transmissions or the time between two consecutive backoff decrements. $E_{p}$ is the payload average length, $P_{t r}$ is the probability that at least one user station transmits in a randomly chosen slot time and $P_{s}$ is the probability of a successful transmission. The detailed derivation of previous parameters can be found in [1].

Several papers have built on this basic model. Wu et al. in [2] extends Bianchi's analysis to include finite retransmission attempts. Q. Ni et al. in [3] and V. Vitsas et al. in [4] adapt the model to backoff mechanism variants. V.M. Vishnevsky et al. in [5], P. Chatzimisios et al. in [6] and T. Nadeem et al. in [7] introduce error probability to the model. T.C. Hou et al. in [8] include the hidden terminal problem. In addition to the throughput analysis, some of the above mentioned papers provide also a companion derivation of the average transmission delay performance.

\section{A. Adjacent-channel Interference}

Adjacent channel interference is due to transmissions in partially overlapping channels. According to [9], the effect of this type of interference produces an SNR degradation and consequently, an increase in the Packet Error Rate (PER). For our evaluation we depart from Chatzimisios' expression for saturation throughput in presence of transmission errors [6]. Chatzimisios et al. redefines $E_{s}$ value of equation (1), defines $P_{e r}$ as the probability a frame is received in error, and includes the PER in $P_{s}$ expression:

$$
\begin{gathered}
E_{s}=\left(1-P_{t r}\right) \cdot \sigma+P_{t r} \cdot P_{s} \cdot T_{s}+P_{t r} \cdot P_{c} \cdot T_{c}+P_{t r} \cdot P_{e r} \cdot T_{e r} \\
P_{e r}=\frac{n \cdot \tau \cdot(1-\tau)^{n-1}}{1-(1-\tau)^{n}} \cdot P E R \\
P_{s}=\frac{n \cdot \tau \cdot(1-\tau)^{n-1}}{1-(1-\tau)^{n}} \cdot(1-P E R)
\end{gathered}
$$

where $n$ is the number of contending stations, $\sigma$ is the duration of an empty slot, $T_{s}, T_{c}$ and $T_{e r}$ are the average time intervals that the medium is sensed busy due to a successful transmission, a collision or a transmission error, respectively, and $\tau$ is the probability that a station transmits a frame in a randomly chosen slot time [1].

\section{B. Co-channel Interference}

Due to the $C S M A / C A$ mechanism, co-channel interference results in undesired effects, such as the hidden node and exposed node problems (cf. [10]). Bianchi's model was again revised to incorporate co-channel interference but authors in [11] only considered two interfering cells. In [12] a complete model is provided whose complexity hinders its implementation as part of a dynamic RRM mechanism. In our approach, the throughput of a WLAN in the presence of cochannel interference is modeled assuming a long term fairness among the nodes sharing the same channel. That is, in the long term, all stations have the same probability to win the contention for the access to the common channel. Although this assumption ignores observable phenomena that arise in various topologies (e.g. see [13]), we have found that the error introduced with individual flow calculations is not critical for the estimation of the global capacity, as it is shown in this section.

In infrastructure mode, given a set of nodes $N$, the algorithm computes the available throughput for every node $i$ $\in N, S_{i}$ as:

$$
S_{i}=s\left(i, C_{i}\right) \cdot \operatorname{Sth}\left(C_{i}\right) ; \quad s(x, Y)=\left\{\begin{array}{cc}
\frac{1}{|Y|} ; & 1-\sum u_{j} \leq \frac{1}{|Y|} \\
1-\sum u_{j} ; & \text { otherwise }
\end{array}\right.
$$

where $C_{i} \subset N$ is the subset of the elements of $N$ that compete for the channel with $i$. This subset $C_{i}$ depends not only on the nodes associated with $i$ 's AP, but also on the co-channel stations from other cells that are within the carrier sense range of $i$, thus we include the influence of exposed nodes. The hidden node problem is minimized with the use of RTS/CTS. Although some error is introduced, we found that the number 


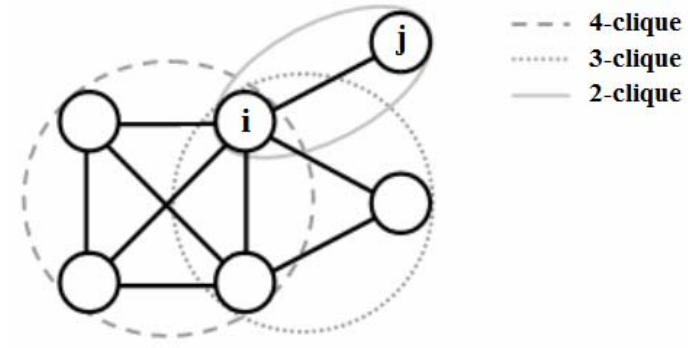

Fig.1: Interference graph and competing stations for node $i$ : $4(C L) ; 6(C R)$

of competing stations for $i$ can be well approximated by the maximum value between the number of nodes in $i$ 's cell and the maximum interference clique to which $i$ belongs. Plotting stations and their interference as an undirected graph, a clique is a subset of nodes such that every two nodes are interferers. This method to model co-channel interference (henceforth $C L$ model) involves the resolution of the clique problem, which is $N P$-complete; if only the number of stations in range is taken into account $(C R)$, the approximation we obtain is good only for lower densities. The differences between $C L$ and $C R$ are illustrated in Fig. 1. The function $\operatorname{Sth}\left(C_{i}\right)$ computes the saturation throughput for the set $C_{i}$, according to (1) and the revisions of [6]. Finally, the multiplier $s\left(i, C_{i}\right)$ is used to reflect the effective share available to $i$. If the utilization of the rest of the contenders is above $\left|C_{i}\right|^{-1}$, the long term fairness will assure a share $s\left(j, C_{i}\right)=\left|C_{i}\right|^{-1} \forall j \in C_{i}$. Otherwise, the excess of bandwidth not used by the members of $C_{i}$ is available to $i$. This can be understood studying the example of fig. 1: following both models $(C L$ or $C R)$, node $j$ will get ideally $1 / 2$ of the maximum throughput (ignoring collisions, errors and other effects to simplify the example), since there is only one competing station: $i$. But actually, $i$ competes with other four nodes, so it will only have at most $1 / 4$ of share. Therefore in practice, $j$ is only competing with $1 / 4$ of a node, thus getting $3 / 4$ of the maximum throughput. The value $u_{j}$ represents the node $j$ 's utilization; but in saturation, $u_{j}$ $=S_{j} / \operatorname{Sth}\left(C_{j}\right)$. Note that $S_{j} \forall j \in C_{i}$ are required to obtain $S_{i}$, i.e. to obtain $S_{i}$, we need to know the value of $S_{i}$ in advance. In order to avoid this incongruity and minimize the error we introduce, a simple heuristic is followed: $S_{i}$ is computed starting with the node having the major number of competitors (higher degree), and so on.

This co-channel model has been evaluated through extensive NS-2 simulations [14]. Note that wireless communication in NS-2 uses different and independent channel objects for the different cells, so then cross-channel noise and interference are not simulated. To fix this issue, our simulation consisted of ad-hoc nodes using only one channel object: four fixed stations played the role of APs and were located so that their coverage ranges partially overlap; the rest of the nodes were randomly placed in the area and were configured to send data to the closest "AP". All these $802.11 \mathrm{~b}$ nodes are driven to saturation by setting up a constant bit rate (CBR) UDP source so that there is always a 1500 byte datagram ready for transmission in every node's queue. So as to isolate the effect of co-channel interference, no transmission errors are introduced. The results of the simulations are compared with both models $(C L$ and $C R)$ in Fig. 2. Even though our calculation of individual flows clearly introduces some error since the starvation problems measured in [15] are not taken into account, Fig. 2 shows that the measure of the overall capacity is balanced and that the error is reduced as the number of nodes is increased. Also note that these approximations are only valid for infrastructure mode, ad hoc schemes usually requires that nodes cooperate to forward each other's packets through the network. This means that the throughput available to each single node's applications is limited not only by the raw channel capacity, but also by the forwarding load imposed by distant nodes [16].

\section{MODELING OF A LINK ADAPTATION ALGORITHM}

IEEE 802.11 standards define several sets of modulations and coding rates for the different physical layers. For example, IEEE $802.11 \mathrm{~b}$ specifies four modes: $11 \mathrm{Mbps}$ (8-bit CCK), 5.5Mbps (4-bit CCK), 2Mbps (DQPSK) and 1Mbps (DBPSK) to be used in the $2.4 \mathrm{GHz}$ frequency band. Each different scheme provides a different transmission rate, but the higher the chosen rate, the worse it performs in the presence of noise and interference; i.e. given certain channel conditions, there is an optimal modulation that maximizes the throughput. As stated in [17], an ideal link adaptation scheme should keep track of the SINR in reception, but this value is unknown for the transmitter, unless a kind of communication between transmitter and receiver is implemented. The authors of [18] presented the Receiver-Based Auto-Rate (RBAR) protocol which proposes modifications in the IEEE 802.11 standard MAC in order to allow the exchange of information regarding channel conditions by means of RTS/CTS messages: first, the receiver estimates the wireless channel quality at the end of the RTS reception, then selects the appropriate transmission rate based on this estimate and feeds back to the transmitter using the CTS. A different approach is based on heuristics and estimations carried out locally in the transmitter side: the Auto-Rate Fallback (ARF) protocol [19] keeps track of a timing function and missed $A C K s$; in [20], the physical rate is adapted to the current link conditions as perceived by the transmitter through received signal strength measurements. In [21] a combination of these two approaches, missing $A C K$ s and measured signal strength, is proposed: Hybrid Auto-Rate Fallback (HARF).

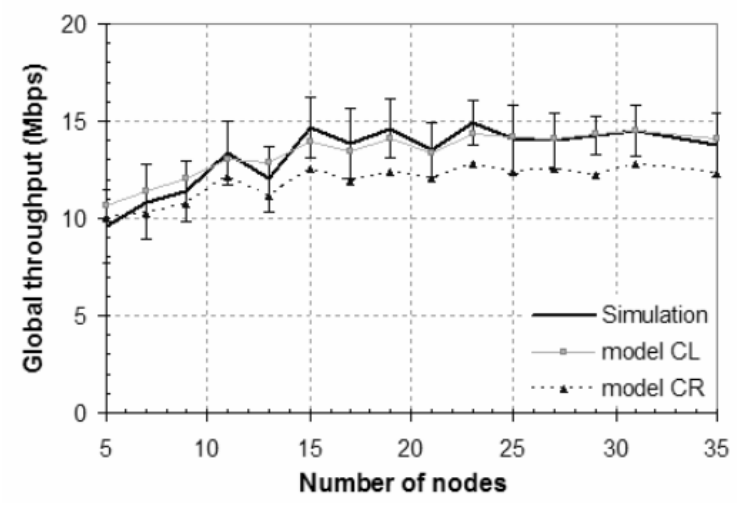

Fig.2: Evaluation of throughput models with co-channel interference through simulations. Model $C L$ obtained by solving maximum clique; model $C R$ obtained by counting co-channel nodes in range 

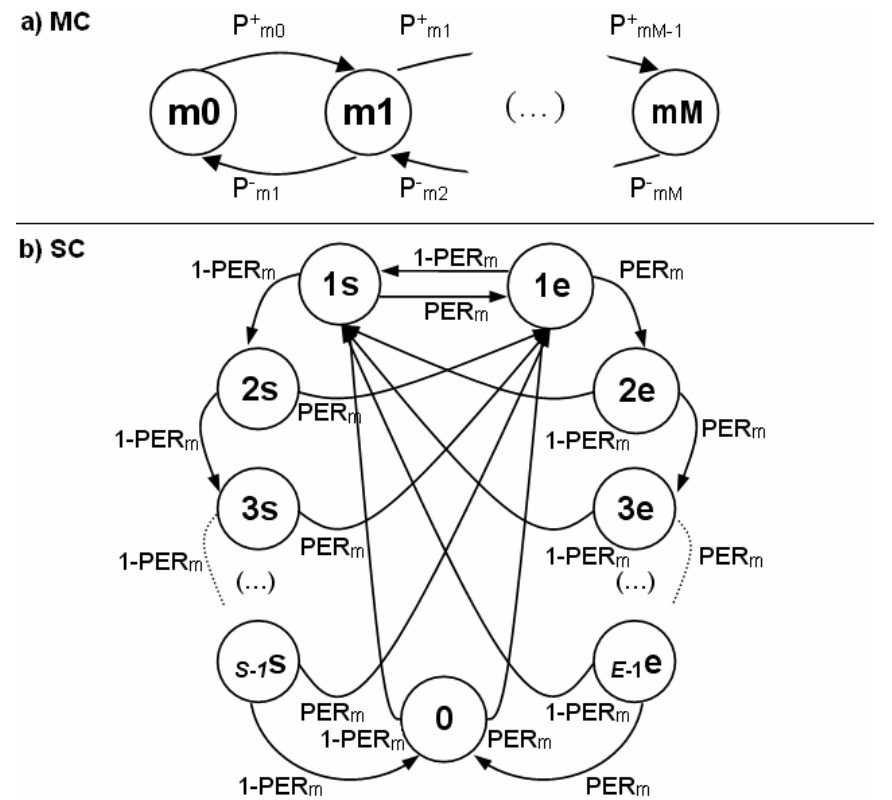

Fig.3: a) MC: Transitions between physical layer modes; b) SC: Markov chain embedded at each MC state.

Most of the IEEE 802.11 MAC implementations have their own proprietary link adaptation schemes, but they are mainly based on heuristics inspired by the original $A R F$ even though it is well known that these schemes cannot react quickly when the wireless channel conditions fluctuate. The fact that these algorithms are not available to the public hampers the inclusion of rate adaptation in realistic analytical throughput models. For these reasons, the main objective of this section is to obtain a theoretical model for a generic rate adaptation algorithm, representative of real life implementations. To this end, in this section we study the behavior of a simple algorithm that counts successful/erroneous consecutive frame transmissions: after $S$ consecutive correct transmissions, the rate is increased and after $E$ consecutive erroneous transmissions, the rate is decreased. This behavior is easy to model and simplifies the resulting formulation, while keeping our results representative of many rate adaptation implementations. The main difference with known implementations is the presence of a timer. In collision prone channels [22], missing $A C K$ s can also be produced by collisions: even if channel conditions are good and the current modulation is optimal, the rate can be reduced due to collisions; the mode reduction leads to higher transmission times thus rising collision probability. The use of timers in acknowledgment-based heuristics is intended to avoid rate reductions as a result of collisions. The absence of a timer in our model is justified by the fact that collisions are not taken into account in the rate selection.

The effect of collisions on throughput performance is already included in original Bianchi's model (cf. III). Recall that the proposed algorithm is intended for modeling purposes and not for a practical implementation although it would render a good performance under low collision probability.

The rate used to transmit any frame in a system with $M$ modes can be modeled by the combination of the Markov chains shown in fig. $3 a$ (main chain, $\mathrm{MC}$ ) and $b$ (secondary chain, SC). Both chains are actually coupled but are studied separately to simplify the resulting formulation. In $\mathrm{MC}$, for any mode $x$, the transitions $P_{m x}^{-}$and $P^{+}{ }_{m x}$ between adjacent states represent the rates of change to mode $x-1$ or $x+1$ respectively. These rates are given by solving the lower level chains (SCs). There is a SC embedded in all $m x$ states of MC. Within SC, after $h$ consecutive transmission errors, state $h e$ is reached; the system reaches any $k s$ state after $k$ consecutive successfully transmitted frames. State (0) of mode $x$ is used as starting point and as a link state between adjacent modes: starting with mode $x$, state (0) of mode $x+1$ is reached after $S$ consecutive transmissions; after $E$ consecutive erroneous frames, the system switches to state (0) of mode $x-1$.

If $P_{s t}$ represents the probability of occurrence of state $s t$, the previously mentioned $P_{m x}$ and $P^{+}{ }_{m x}$ will be $P_{(E-1) e} \cdot P E R_{m}$ and $P_{(S-1) s} \cdot\left(1-P E R_{m}\right)$ respectively. Note that the $P E R_{m}$ is different for each modulation $m$ given the SINR; and that computation of stationary probabilities is not affected by the fact that the Markov renewal process is actually embedded at slot boundaries. By solving SC, the probabilities of the different states can be written as a function of $P_{l e}$ and $P_{l s}$, as follows:

$$
\begin{gathered}
P_{1 e}=\operatorname{PER}_{m}\left(P_{0}+\sum_{i=1}^{S-1} P_{i s}\right) \\
P_{1 s}=\left(1-P E R_{m}\right)\left(P_{0}+\sum_{i=1}^{E-1} P_{i e}\right) \\
P_{h e}=P_{(h-1) e} P E R_{m}=P E R_{m}^{k-1} P_{1 e} ; 1<h<E \\
P_{k s}=P_{(k-1) s}\left(1-P E R_{m}\right)=\left(1-P E R_{m}\right)^{k-1} P_{1 s} ; 1<k<S \\
P_{0}=\left(1-P E R_{m}\right)^{S} P_{1 s}+P E R_{m}^{E} P_{1 e}
\end{gathered}
$$

Note that $P_{0}$ actually depends on $P E R_{m-1}$ and $P E R_{m+1}$, but in (10) it appears as a function of $P E R_{m}$. This error is due to the simplification introduced by the decoupling of the chains. However, this error is always smaller than 1\% (compared with simulations in which $10^{6} 1000$-Byte packets are sent).

The sum of all probabilities $\left(P_{0}, P_{k s}, P_{h e} \forall k<S\right.$ and $\left.h<E\right)$ must be 1 . From the relation between $P_{l e}$ and $P_{l s}$ depicted in (11) and derived from (6) to (10), we can leave all probabilities as a function of $P E R, S$ and $E$ :

$$
\begin{gathered}
P_{1 s}=P_{1 e} \frac{\left(1-P E R_{m}^{E}\right)}{1-\left(1-P E R_{m}\right)^{S}} \\
P_{1 e}=\left(\frac{1-P E R_{m}^{E}}{1-\left(1-P E R_{m}\right)^{S}} \sum_{i=0}^{S-1}\left(1-P E R_{m}\right)^{i}+\sum_{i=0}^{E-1} P E R_{m}^{i}\right)^{-1}
\end{gathered}
$$

Once we obtain the transition probabilities for all modes $\left(P_{m x}^{-}\right.$and $\left.P_{m x}^{+}\right)$, the resolution of the chain $\mathrm{MC}$ is straightforward and allows us to compute the average PER and the average transmission rate as a weighted sum:

$$
\begin{gathered}
P_{m 0}=\left(1+\sum_{i=1}^{M}\left(\prod_{j=1}^{i} \frac{p_{m(j-1)}^{+}}{p_{m j}^{-}}\right)\right)^{-1} \\
P_{m x}=P_{m 0} \prod_{j=1}^{x} \frac{p_{m(j-1)}^{+}}{p_{m j}^{-}} ; 0<x \leq M
\end{gathered}
$$




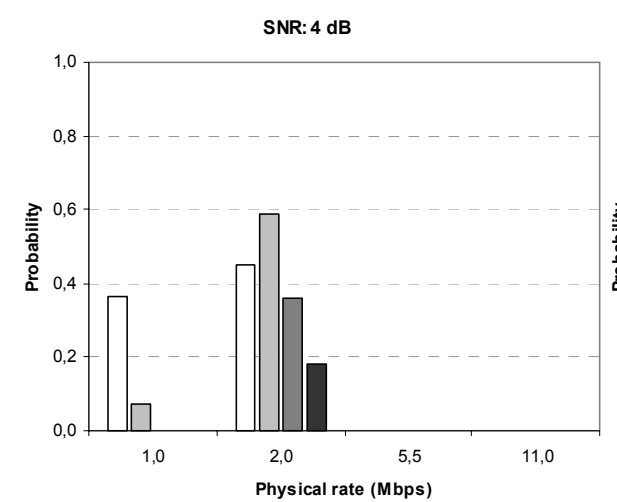

$\mathrm{E}=2 \mathrm{~S}=10$

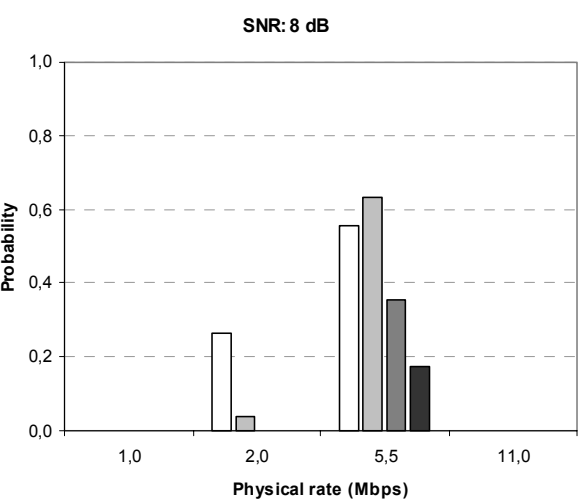

$E=6 S=3$
SNR: $12 \mathrm{~dB}$

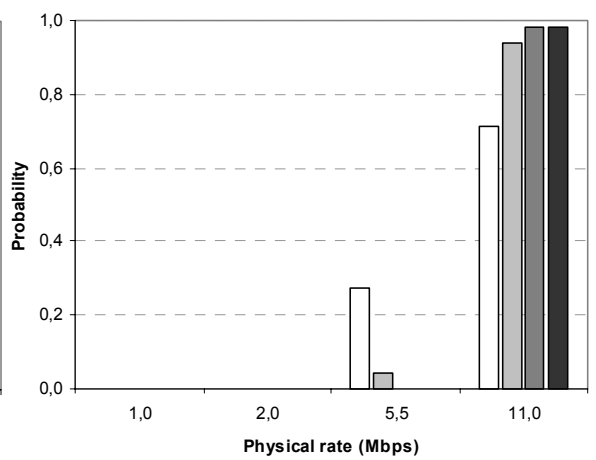

$E=10 S=2$

Fig. 4. Histogram of successful transmitted packets for different $S N R$ values and different $(E, S)$ combinations

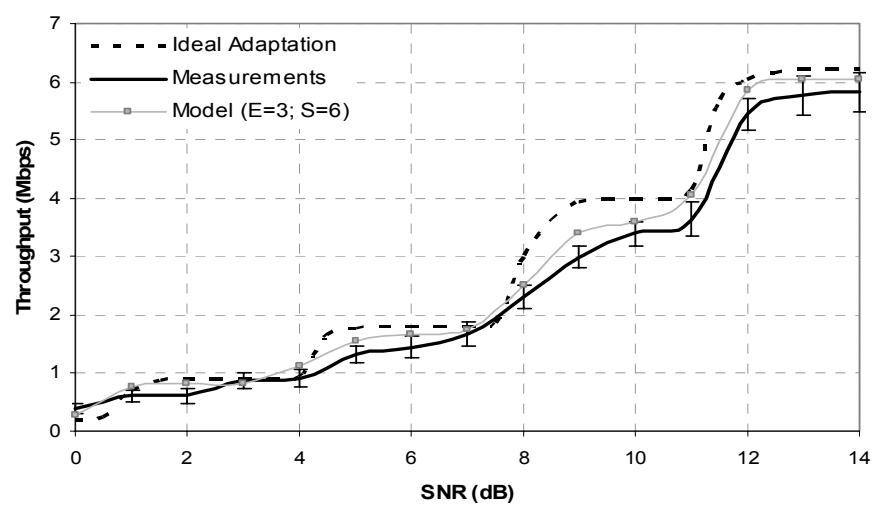

Fig. 5. Rate adaptation model performance and real measurements.

$$
P E R=\sum_{i=0}^{M} P E R_{m i} P_{m i}
$$

Using these values in equations (1) to (4), as explained in section III, we can include the effect of rate adaptation into the saturation throughput model (equation (5)).

The best choice of $E$ and $S$ parameters has been settled after evaluating the model under different values of PER. Figure 4 is a sample of those tests: the three figures represent the number of packets successfully transmitted (normalized by the total number of transmitted packets) by a single IEEE 802.11 b station operating at a given modulation and using different combinations of $S$ and $E$. As it was expected, with high signal quality, the best performance is obtained with small values of $S$ (and a bigger $E$ ). On the other hand, with a high loss rate, it is preferable to have a small $E$ (and a bigger $S$ ). This trade-off is solved by selecting $E=3$ and $S=6$, which provides a good performance for a wide range of $S N R$.

\section{Practical Evaluation}

In order to evaluate the reliability of the rate adaptation algorithm modeled in Section IV, the goal of the first set of tests was the comparison of the expected performance of the algorithm with measurements obtained in a real scenario. The scenario consists of two laptops equipped with Intersil Prism IEEE 802.11 b WLAN cards configured in automatic rate selection. Prism cards implement a rate adaptation algorithm [23] similar to $A R F$ : rate fallback is triggered by transmit retries; a timer function and successful transmissions increase

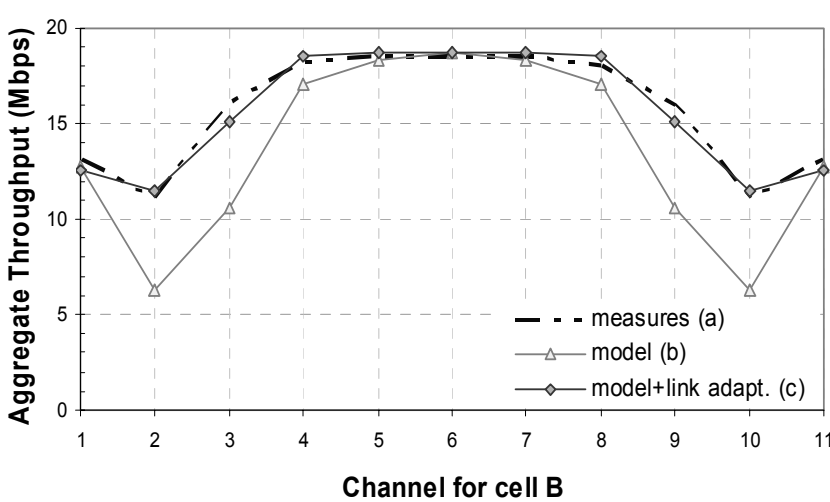

Fig.6: Aggregate throughput for cells $A, B$ and $\mathrm{C}$ for different channel configuration in cell $B$

rate. One CBR UDP connection is set to send 1500 Byte packets. Samples of throughput and $S N R$ are taken while the distance between the laptops is slowly increased.

As shown in fig. 5, the mean throughput values obtained with practical measurements are lower than expected. This is not only due to the use of different rate adaptation algorithms. Indeed, taking measurements in a real testbed involves facing undesirable effects from environmental factors that negatively affect the measured throughput. While the analytical model only takes thermal noise into account, interference from other systems could have affected the testbed performance. Moreover, SNR measurements provided by the wireless device firmware can be inaccurate. However, the values provided by the model can be considered as an admissible upper bound, which is most of the time within one standard deviation away from the mean of measured samples. The dotted line represents the upper bound that could only be achieved with an ideal adaptation (i.e. instantaneous knowledge of receiver's channel conditions, cf. [20]).

In order to validate the proposed model (5), including both adjacent and co-channel interference, another testbed has been built. Without loss of generality we consider a scenario of three IEEE $802.11 \mathrm{~b}$ overlapping cells $(A, B$ and $C$ ) with two nodes each (AP and client), since it allows the analysis of the combined effect of adjacent and co-channel interference and hence, it remains applicable in larger scenarios. For each client, an upstream UDP source is set as in the previous tests. Channel 1 is set in cell $A, C$ uses channel 11; the aggregate throughput of the three cells is measured for different 
channels of $B$ (1 to 11$)$. The first results obtained with our model using a fixed rate of $11 \mathrm{Mbps}$ correspond to fig. 6 line $b$; real measurements are depicted by line $a$. Once we include rate adaptation (line $c$ ), it is clearly shown that our approach closely models the measured results, even though the rate adaptation used in the model is slightly different from the algorithm actually implemented in the wireless devices.

\section{CONCLUSIONS}

A new method is introduced to obtain the maximum capacity of a multicell IEEE 802.11 network, considering the effect of co-channel and adjacent channel interference. An analysis of a simple method for the link adaptation is also included, which helps to provide more realistic results. In spite of the fact that the rate adaptation algorithm presented is only valid for modeling purposes, its performance has been shown to be representative of real-life devices, which implement proprietary algorithms. In this way, our model remains independent of the hardware used.

Despite the simplicity of the proposed algorithm and the considered assumptions, it has been demonstrated through simulations and real measurements that it provides reasonably accurate estimation of the saturation throughput. Therefore, our model is a helpful tool for the qualitative evaluation of a multicell WLAN scenario. In the future, we plan to introduce this capacity estimation in different RRM schemes, such as channel allocation, power assignments and load balancing algorithms as the objective function that has to be optimized and used to evaluate the possible configurations.

\section{REFERENCES}

[1] G. Bianchi, "Performance analysis of the IEEE 802.11 Distributed Coordination Function," IEEE Journal on Selected Areas in Communications, vol. 18, pp. 535-547, March 2000.

[2] H. Wu, Y. Peng, K. Long, S. Cheng and J. Ma, "Performance of Reliable Transport Protocol over IEEE 802.11 Wireless LAN: Analysis and Enhancement", in Proc. of INFOCOM 2002, June 2002.

[3] Q. Ni, I. Aad, C. Barakat and T. Turletti, "Modeling and Analysis of Slow CW Decrease for IEEE 802.11 WLAN", in Proc. of PIMRC 2003, September 2003.

[4] V. Vitsas and A. Boucouvalas, "Performance Analysis of the Advanced Infrared (AIr) CSMA/CA MAC Protocol for Wireless LANs", ACM Wireless Networks, vol. 9, pp. 495-507, September 2003.
[5] V. M. Vishnevsky and A. I. Lyakhov, "802.11 LANs: Saturation Throughput in Presence of Noise", in Proc. of IFIP Networking 2002, May 2002.

[6] P. Chatzimisios, A. Boucouvalas, and V. Vistas, "Infuence of channel BER on IEEE 802.11 DCF," Electronics Letters, vol. 39, pp. 16871689, November 2003.

[7] T. Nadeem and A. Ashok, "IEEE 802.11 DCF Enhancements for Noisy Environments", in Proc. of PIMRC 2004, September 2004.

[8] T. C. Hou, L. F. Tsao and H. C. Liu, "Throghput Analysis of the IEEE 802.11 DCF Scheme in Multi-hop Ad-hoc Networks", in Proc. of ICWN 2003, June 2003.

[9] E. Garcia, E. López-Aguilera, R. Vidal and J. Paradells, "Effect of adjacent-channel interference in IEEE 802.11 WLANs," in Proceedings of the 2nd International Conference on Cognitive Radio Oriented Wireless Networks and Communications CROWNCOM'07, August 2007.

[10] K. Heck, "Wireless LAN performance in overlapping cells," in IEEE 58th Vehicular Technology Conference, VTC'03-Fall, vol. 5, pp. 28952900, October 2003.

[11] M. K. Panda, A. Kumar, S. H. Srinivasan, "Saturation throughput analysis of a system of interfering IEEE 802.11 WLANs", in Proc. of IEEE International Symposium WoWMoM'05, June 2005.

[12] M. Garetto, T. Salonidis and E.W. Knightly, "Modeling Per-flow Throughput and Capturing Starvation in CSMA Multi-hop Wireless Networks," in Proceedings of the 25th Conference on Computer Communications, IEEE Infocom '06, April 2006.

[13] C. Chaudet, D. Dhoutaut and I.G. Lassous, "Performance issues with IEEE 802.11 in Ad Hoc Networking," in IEEE Communications Magazine, vol. 43 n. 7, pp. 110-116, July 2005.

[14] The Network Simulator - ns-2; http://www.isi.edu/nsnam/ns/.

[15] C. Chaudet, D. Dhoutaut and I.G. Lassous, "Experiments of some performance issues with IEEE $802.11 \mathrm{~b}$ in ad hoc networks," $2^{\text {nd }}$ Annual Conference on Wireless On-Demand Network Systems and Services, WONS'05, January 2005.

[16] Li J, Blake C, De Couto D, Lee HI, Morris M. Capacity of Ad Hoc wireless networks. Proceedings of the 7th ACM MobiCom, pp: 61 69. 2001.

[17] D. Qiao, S. Choi, and K. Shin, "Goodput analysis and link adaptation for IEEE 802.11a wireless LANs," IEEE transactions on Mobile Computing, vol. 1, pp. 278-292, December 2002.

[18] G. Holland, N. Vaidya and P. Bahl, "A rate-adaptive MAC protocol for multi-hop wireless networks," in ACM Mobicom'01, pp. 236-251, July 2001.

[19] A. Kamerman and L. Monteban, "WaveLAN-II: a high performance Wireless LAN for the unlicensed band," Bell Labs technical Journal, pp 118-133, 1997.

[20] J. del Prado and S. Choi, "Link adaptation strategy for IEEE 802.11 WLAN via received signal strength measurement," in IEEE ICC '03, vol. 2, pp. 1108-1113, May 2003.

[21] Y-J. Kim and Y-J. Suh, "An efficient rate switching scheme for IEEE 802.11 wireless LANs," in IEEE 61st Vehicular Technology Conference, VTC'05-Spring, vol. 4, pp. 2364-2368, May 2005.

[22] A. Bazzi, M. Diolaiti and G. Pasolini, "Link adaptation algorithms over IEEE 802.11 WLANs in collision prone channels," in IEEE 63rd Vehicular Technology Conference, VTC'06-Spring, May 2006.

[23] Intersil Corporation, PRISM Driver Programmer's Manual, Version 2.30, June, 2002. (For distribution under NDA only). 